\title{
Embodied Cognition, Perception, and Performance in Music
}

\author{
ANDREW GEEVES[1] \\ Departments of Psychology and Cognitive Science, Macquarie University, Australia \\ JOHN SUTTON \\ Department of Cognitive Science, Macquarie University, Australia
}

\begin{abstract}
In this response to Leman and Maes's paper in this issue, we raise a couple of concerns about the authors' particular approach to embodied music cognition, drawing selectively on their other writings to enrich our interpretation of this target article, while pointing to a few of the many other legitimate research paths that can also fall under this label. We explore two underlying dichotomies implicit in the research programme adumbrated by Leman and Maes - cognition/embodiment and perception/performance - and implications for their theory of embodied music cognition. We then examine research that has focussed on the perspective of the music performer.
\end{abstract}

Submitted 2014 November 12; accepted 2014 November 24

KEYWORDS: embodied cognition, music, music perception, performance

LEMAN, Maes, and their colleagues at IPEM in Ghent have conducted a diverse and rich array of empirical studies of embodied music cognition. Their current short review paper (Leman \& Maes, this issue) sounds out just a few, perhaps somewhat disconnected sequences of notes and phrases extracted from this extraordinary body of work, with a focus on 'the role of embodiment in music perception'. Readers will anticipate and desire more, and will hunt down other versions of these themes and variations (Leman, 2007; Maes, Leman, Palmer, \& Wanderley 2014). We greatly admire both the scientific studies they describe and 'the embodied music cognition theory' (this issue, abstract) with which they are linked. But of course this theory raises further questions, and is not the only theory in this domain.

\section{COGNITION AND EMBODIMENT}

Leman and Maes outline an assumption underlying their embodied music cognition paradigm: gesture and sound exist in a relationship mediated by mirroring processes. In their view, "[E]mbodiment assumes the existence of mirroring processes that facilitate the encoding of expressive gestures into sounds, and the decoding of sounds into expressive gestures" (p. 237). Amongst other gestures that Leman and Maes identify as occurring during live music performance, musicians make movements to accompany communicative expression (expressive-supporting gestures), while audience members react to communicative expression in music by moving in certain ways (expressive-responding gestures). Such gestures, the authors suggest, bestow with meaning human sensorimotor engagement with music.

This approach has driven some wonderfully detailed analyses of expressive gestures in specific musical contexts (Leman 2007, chapter 6). But by partitioning musical gesture in this way, Leman and Maes risk creating a false dichotomy between action and perception that is at odds with the official claims of their embodied music cognition paradigm. This is one of a number of potentially problematic dichotomies which remain at the heart of their approach, and is in some tension with their intention to overcome prior disembodied or dualist views. This embodied music cognition theory has a peculiar starting-point, in the claim that the human body is "a mediator for meaning formation" (this issue, p. 236). Such a mediator is required, according to Leman, because there is a fundamental "gap" between "music as encoded physical energy" and the "mental level in which experiences, values, and intentions form the basic components of musical signification" (Leman, 2007, p. xiii). The body is the mediating vehicle that drives and makes possible the "process of turning physical energy into actionrelevant and, as a consequence, action-intended ontology" (p. 84). In this view, mediation, either by way of body movements and embodied interactions, or by artificial mediation technologies, is necessary because ideas or mental representations have somehow to be transferred "into a material or energetic form" (p. xiii, pp. 71-76). We agree with other critics that there is a danger here of allowing a "dualistic bias" back into a putatively "embodied" theoretical paradigm (Matyja, 2012; Schiavio \& Menin, 2013). The insistent usage of the terms "embodied" and "embodiment" in some areas of 
cognitive theory may sometimes, as Maxine Sheets-Johnstone (2009) argues, signal residual difficulties in accepting that there is no gap of this kind to be bridged, that animated organic matter of certain kinds in certain settings is already alive and feeling, moving and motivated, engaged and thinking, expressing and responding. Within a thoroughgoing and exuberant or visceral materialism, ideas and meanings and experiences are already "in material or energetic form" (Sutton \& Tribble, 2012). Leman and Maes do also offer more unambiguous statements of materialism, and clearly accept the need to study the microtemporalities of affective-kinetic coordination dynamics on which SheetsJohnstone urges us to focus. But, we suggest, we can still see in their work a number of subtler traces of their initial and jarring conceptualization of cognition as distinct from embodiment.

In underlining their appropriate emphasis on the dynamic nature of music perception and cognition, Leman and Maes (this issue, p.241) say that "Music perception and cognition encompass different systems, such as the auditory system, motor system, affective system, and cognitive system (e.g., meta-knowledge about a musical piece, autobiographic memories, etc.) situated within a specific environmental context". While they go on to stress that interactions between these systems are vital, this confirms that they are indeed treating cognition as fundamentally distinct from sensation, the motor system, emotion, and the environment. Elsewhere we see that Leman and Maes retain a narrower, higher-level conception of cognition, which divides it sharply from more bodily aspects of our engagement with music. They assume that if it turned out that music perception was based on lower-level sensory processes rather than involving long-term memory, "then music perception would not be linked with cognitive processes, but rather with sensory processes", as if the realms of the cognitive and the sensory are entirely mutually exclusive (Leman \& Maes, 2014, p. 82). Likewise, if our perception of musical tempo relies heavily on "inherent constraints" provided by the environment and by the body, such as acoustical constraints or biomechanical resonators, these factors would form "a reference outside the cognitive system" (p. 83). These points contrast curiously with their strong criticism elsewhere of "classical" views on which perception and action "are outside central cognition" (Maes et al., 2014, p. 1). But even in the course of the resulting suggestive discussion of the role of complex dynamic interactions in musical expressivity, they again paradoxically describe the body as "a mediator between sensory and motor processes and mental states" (Maes et al, 2014, p. 9), which suggests again that they treat mental states as distinct from both the body and sensory-motor processes. For sure, they do distance themselves from theorists who seek to explain music perception in terms of internal cognitive processes alone, and they stress that music perception involves interaction between cognition, on the one hand, and body or environment, on the other, such that it is "not detached" from such "environmental constraints and particularities of the human body" (Leman \& Maes, 2014, p. 83). But since such interaction is only possible between systems which are in themselves distinct, such constant references to dynamics only serve to prove that Leman and Maes remain committed to a fundamental gap between cognition and sensation or embodiment.

This point also seems at odds with Leman and Maes's claim that their embodied music cognition theory is "closely linked" to the hypothesis of extended cognition (this issue, p. 236). Leman and Maes certainly stress the range of ways in which music perception and cognition depend heavily on external resources, and it is of great importance genuinely to turn the attention of a scientific field towards the detailed study of the embedded or deeply scaffolded nature of cognition (Sterelny, 2010; Sutton, Harris, Keil, \& Barnier, 2010): but still, this is different from the stronger claim that these processes can in certain circumstances be literally distributed across or constituted in part by such external processes (Cochrane, 2008; Krueger, 2014). We would very much like to see embodied music cognition theory move further in the direction of Krueger's account of the "musically extended mind", in which musical affordances solicit and enhance complex processes of emotion-regulation and experiences not otherwise available to us (Krueger, 2011, 2014). Such an account requires theorists to examine in depth "concrete instances ... of how we use music in everyday life" to develop and engage in complex forms of affective synchrony (Krueger, 2014, p. 6). In this way, such an account is also arguably also closer to ethnographic studies of music such as those we discuss below, or to historical work in carnal musicology (Le Guin, 2006), or to studies of historical changes in the distributed cognitive ecologies of music and song (Tribble \& Keene, 2011, chapter 4). In contrast, the current version of Leman and Maes's version of embodied music cognition theory can occasionally appear somewhat bloodless in its distance from the natural and social settings of musical interaction in the wild.

We briefly note two further manifestations of these uneasy residual dualisms. Leman's claim that modern technologies profoundly reorganize our musical experience is based in part on his distinction between two kinds of constraints on our musical activity. Natural constraints, he argues, "subsume the laws of physics and biology, whereas cultural constraints are rules or conditions that impose limits on what is acceptable, appreciated, and considered to be true or valid in a culture" (Leman, 2007, p. 55). Again, Leman claims that there are rich interactive relations between natural and cultural constraints, and that through action we unify these distinct constraints, transforming physical energy to the cultural level and vice versa: but again, this is a peculiar and dichotomous starting-point 
for a theory of embodied cognition. In stark contrast, the framework of extended or distributed cognition developed by Clark (1997) and Hutchins $(1995,2010)$ can be plausibly interpreted as entirely dismantling the barrier between nature and culture in the realm of human cognition and intelligent embodied activity (Sutton, 2010). If we are "natural-born cyborgs", whose uniquely plastic brains create, adapt, and incorporate cultural norms, symbols, and tools which we ourselves have constructed, then our human cultures are themselves profoundly natural, such that there is, once more, no gulf here to be bridged (Donald, 1990; Clark, 2000, 2003; Christensen \& Sutton, 2012; Sterelny 2012).

Secondly, Leman distinguishes two forms of understanding or intentionality involved in our practices of musical signification. "Corporeal intentionality", on the one hand, involves "the articulation of moving sonic forms, with the emphasis on movement in relation to behavioural resonances of the human body" (2007, p. 84). He sees such corporeal understanding as based "on mimetic processes originating in motor resonance processes" (p. 237), as also sketched briefly in the current review paper: these "corporeal articulations" are the topic of much of the most innovative empirical research at IPEM. In contrast, "cerebral intentionality" is "based on a cognitive interpretation of subjective experiences and on the projection of these experiences onto cultural contexts": its "essence ... is interpreting the source of intentions attributed to music" (p. 84, p. 237). Again, Leman argues that both corporeal and cerebral understanding are vital, and also that they interact: but they are initially conceived as fundamentally distinct. Relatedly, he distinguishes a non-cognitive form of corporeal immersion in music as sound energy, from a form of awareness which involves knowledge, reflection, evaluation, and interpretation. He argues that "awareness and immersion have to be balanced" (2007, p. 6). Again the starting-point here is unnecessarily dualist. We can compare dichotomies between intuition and reflection across related theoretical contexts such as moral cognition, absorbed coping, skilled movement, and expert decision-making. In each case it is not enough to adopt a traditional, firm dichotomy between mindless intuitive processes and intelligent strategic processes but then stress that these two sets of processes operate in complex interactions with each other (Bortolotti, 2011). Rather, in each case, we have argued on the basis of a range of conceptual and empirical grounds, the initial idea that there is a clear distinction between two unified sets of processes should be jettisoned (Sutton, McIlwain, Christensen, \& Geeves, 2011; Christensen \& Sutton, 2012; Geeves, McIlwain, Sutton, \& Christensen, 2014). If the realm of immersed embodied intentionality is already shot through with intelligence, then corporeal articulations and understandings do not have to be balanced against more aware "cerebral" understandings.

\section{THE PERCEPTION-PERFORMANCE DICHOTOMY}

Leman and Maes describe a number of empirical studies of live music performance conducted at IPEM that have "aimed at better understanding the sensorimotor basis for encoding expression in music" (this issue, p. 237). Taking the music listener as their subject, these studies demonstrate that audience members mimic some of the gestures involved in the production of the live music performance that they observe. However, these studies neglect to take fully into account the experience of the music performer, tending to focus instead on the perspective of the music listener or to home in on a particular element of live music performance for a musician. In the context of live music performance, music listeners' perception of music is inextricably linked to music performers' production of music. For example, from the perspective of the music performer, what might be the relationship between the "gesture alphabet" that Leman and Maes suggest musicians possess and the finding they cite that music listeners will mimic the expressive, but not sound-producing, gestures of a performer? Might a musician attempt to gain the attention of a particularly unresponsive audience by incorporating into his/her performance a greater number of more exaggerated expressive gestures? To what extent, if any, might the way in which music listeners receive and possibly mimic performers' gestures affect the range of the gesture alphabet that is available to a music performer during a particular live music performance? In other words, during live music performance, to what extent might expressivesupporting and associated gestures be open to change, manipulation and influence on account of musicians' levels of expertise? What types of gestures are exchanged between music performers, and what relationships do these share with the gestures that music listeners might mimic in their perception of live music performance?

That such an account of looping feedback between performative expression and receptive expression (and back) is missing from the studies profiled in Leman and Maes' article is curious given that Leman, in his (2007) model of social music communication, argues that any actions made on stage influence listeners' response to music, which then influences the actions produced on stage. In his ethnographic study of jazz musicians, Berliner (1994) uses the metaphor of conversation to capture this kind of reciprocal relationship, a relationship he observes occurring between audience members and musicians during a jazz performance: "Performers and listeners form a communication loop in which 
the actions of each continuously affect the other", he writes (p. 459). In our research exploring the experience of music performance for the professional musician, Emily, a professional musician, describes a similar type of cumulative conversation that she experienced as developing between her and an audience that were particularly engaged with her performance: "The audience is so responsive. They give you a chunk of stuff and that lets you construct your song with that energy...you give it back.... and then they build and build. We're all building together. It's like a convection current of energy" (Geeves, Mcllwain \& Sutton, in press). Evidence such as this points to the interrelatedness of musicians' and listeners' experiences of live music performance and speaks to the need to take into equal consideration the voices of the music listener and the music performer when attempting to gain a comprehensive understanding of happenings that occur in the context of live music performance.

The reciprocal nature of the exchanges that transpire between music listeners and performers during live music performance needs to be taken into account by studies that aim to better understand the process of encoding and decoding expression in music and its sensorimotor basis. By too strongly separating encoding from decoding processes - by creating a false dichotomy between action and perception, even with the intention of later linking the two again - Leman and Maes divorce music perception from music production. By focusing more on the music listener than the music performer, they risk obtaining a half-complete picture of the phenomena that are of interest to them. This is true especially when considering the relationship between gesture and sound in live music performance, as this relationship is embedded in the interaction of performing musicians with an audience and of music perception with music performance. Future research within the embodied music cognition paradigm and associated areas would benefit from considering music perception in the broader context of music performance, expanding the focus of previous studies on a particular element of and perspective within music performance to a more gestalt understanding of performance.

\section{THE EXPERIENCE AND VOICE OF THE MUSIC PERFORMER}

Here, we provide two examples of research that has taken into consideration the perspective of the music performer in its examination of music performance. The first is from work conducted by Clayton and colleagues in the "Experience and Meaning in Music Performance" (EMMP, 2005-8) programme (Clayton, Dueck, \& Leante, 2013). The researchers combined audio, visual, and participant observation and interview data gathered from live music performance. In two ethnographic studies, Clayton (2007a, 2007b) examined the gestures that musicians shared with each other in North Indian rag performance. In the first, Clayton (2007a) found that, over and above the use of eye contact and bodily orientation, musicians gestured to each other during performance to share the experience of time and motion. Clayton named gestures related to the content of the singing in the performance as "Illustrators" while gestures related to music process and structure were named "Markers". Secondly, Clayton (2007b) studied a performance by vocal and tanpura (plucked lute) soloist Veena Sahasrabuddhe in which she was accompanied by one harmonium, one tabla and two tanpura players. Clayton found that every time one of the tanpura accompanists fixed her visual attention on Sahasrabuddhe's back, her movements would become coordinated with those of the soloist. The accompanist was unaware of this occurring and actively sought to keep the rhythm of her tanpura separate from that of the other instruments. That such synchronised movement between two musicians can occur outside conscious awareness and despite explicit efforts to resist it is presented by Clayton as evidence for the strength of entrainment processes in music performance.

Leman and Maes (this issue, pp. 239-240) also address studies of entrainment, and present the existence of entrainment as lending support to their theory of embodied music cognition. However, Leman and Maes do not make the links between entrainment and embodied music cognition clear. They neither provide a working account of their understanding of entrainment nor explain how it supports an embodied understanding of music cognition. Entrainment is "the process by which independent rhythmical systems interact with each other" (Clayton, 2012, p. 49). In some cases "they adjust towards and eventually 'lock in' to a common phase and/or periodicity" (Clayton, Sager \& Will, 2005 p. 2), though in other cases, especially in live music performance, entrainment will involve independent rhythmical systems interacting in ways other than just locking in together in this way. Leman and Maes argue that understandings of entrainment should be expanded so that entrainment is understood as a spatiotemporal, rather than just temporal, phenomenon. To back up their claim, they profile one study that examined the walking pace of subjects exposed to musical excerpts with identical tempi but different musical expression and one study that measured children's capacity to tap along with a musical beat.

Leman and Maes do not specify how the results of these studies support their assertion that entrainment in music performance is a spatiotemporal phenomenon. Missing from their account is a clear explanation of this link, and examples of entrainment operating across time and space in realworld live music performance. Leman and Maes assert the novelty of their claim that entrainment is a 
spatiotemporal phenomenon. It is true that some researchers' definitions have emphasised entrainment as based primarily on a timing dimension (Clayton, 2012; see also Leman's [2012] response to Clayton): but other existing views do characterise it as a spatiotemporal phenomenon. In addition to studies of the basic entrainment processes underlying mechanisms of joint action and cohesion in music performance (Keller, 2008, 2014; Phillips-Silver and Keller, 2012; Keller, Novembre \& Hove, in press), we can point to Doffman's ethnographic work with jazz musicians $(2009,2011,2013)$. Doffman offers rich, experience-near examples of the ways in which entrainment allows musicians to establish "groove" with each other during live performance and to coordinate both their actions within the realm of space and rhythms within the domain of time. While initial definitions of entrainment may have focused on its operation in the domain of time, there is no shortage of more recent research that views entrainment as a spatiotemporal phenomenon.

Our second example of research that takes into consideration the perspective of the music performer addresses entrainment in time and space during live music performance. As we describe in greater detail elsewhere (Geeves, McIlwain \& Sutton, 2014), we take as a case study a moment during live music performance in which entrainment amongst a musical quartet appears to be threatened. At this moment, the lead member of the ensemble improvises and expands the structure of the last chorus of a piece of music beyond that which has previously been rehearsed (during four lengthy rehearsals) and performed live (more than ten times during two different Australian tours). The other three musicians in the quartet follow the leading performer and smoothly transition into unprecedented performance territory. Our paper examines the mechanisms, consolidated over previous rehearsals and performances, which allow the other ensemble members to accomplish this unanticipated feat so smoothly. The data that form this case study were taken from intensive fieldwork, video and audio data and semi-structured interviews conducted with the four musicians over an extended period. Tracing the evolution of the structure of this piece of music from the ensemble's first rehearsal together to the case study performance and beyond, we find evidence of a sense of trust established amongst the four musicians via entrainment processes, improvisatory strategies, and use of eye contact and gesture. We suggest that this trust allows the musicians to use the threat of a break in entrainment processes in a performative manner, serving to entertain and engage their audience.

We present these examples of research that starts from the perspective of the music performer as complementary to the material discussed by Leman and Maes. Ethnographic work such as that conducted by Clayton (2007a, 2007b), Doffman (2009, 2011, 2013), and ourselves (Geeves, McIlwain \& Sutton, 2014) exemplifies research on live music performance with high ecological validity, and is highly relevant to concepts of interest in the embodied music cognition paradigm. Leman and Maes suggest that their "empirical findings demonstrate that embodiment is only one component in an interconnected network of sensory, motor, affective, and cognitive systems involved in music perception" (this issue, abstract, p. 236). We suggest, in contrast, that embodiment, in the sense of the ongoing engagement of the affective-tactile-kinesthetic body (Sheets-Johnstone 2009), might be seen not as a single and separable interactive component of our musical, perceptual, and cognitive capacities, but rather as an entirely integrated and pervasive dimension of them.

\section{ACKNOWLEDGEMENTS}

Many thanks to Simon Høffding and Doris McIlwain for comments on an earlier draft, and to Kevin Ryan for assistance and support.

\section{NOTES}

[1] Departments of Cognitive Science and Psychology, Macquarie University, Balaclava Road, North Ryde, NSW 2109 AUSTRALIA. andrew.geeves@mq.edu.au

\section{REFERENCES}

Berliner, P. F. (1994). Thinking in jazz: The infinite art of improvisation. Chicago, IL: University of Chicago.

Bortolotti, L. (2011). Does reflection lead to wise choices? Philosophical Explorations, 14, 297-313.

Christensen, W., and Sutton, J. (2012). Reflections on emotions, imagination and moral reasoning: towards an integrated, multidisciplinary approach to moral cognition. In R. Langdon \& C. Mackenzie (Eds.), Emotions, Imagination, and Moral Reasoning (pp. 327-347). London: Psychology Press. 
Clark, A. (1997). Being there: Putting brain, body, and world together again. Cambridge, MA: MIT Press.

Clark, A. (2000). Word and action: Reconciling rules and know-how in moral cognition. Canadian Journal of Philosophy, S26, 267-289.

Clark, A. (2003). Natural-born cyborgs. Oxford: Oxford University Press.

Clayton, M. (2007a). Time, gesture and attention in a "Khyāl" performance. Asian Music, 38, 71-96.

Clayton, M. (2007b). Observing entrainment in music performance: Video-based observational analysis of Indian musicians' tanpura playing and beat marking. Musicae Scientiae, 11, 27-59.

Clayton, M. (2012). What is entrainment? Definition and applications in musical research. Empirical Musicology Review, 7, 49-56.

Clayton, M., Dueck, B., \& Leante, L. (2013). Experience and meaning in music performance. Oxford: Oxford University Press.

Clayton, M., Sager, R., \& Will, U. (2005). In time with the music: The concept of entrainment and its significance for ethnomusicology. European Meetings in Ethnomusicology, 11, 3-142.

Cochrane, T. (2008). Expression and extended cognition. Journal of Aesthetics and Art Criticism, 66, 329-340.

Doffman, M. (2009). Making it groove! Entrainment, participation and discrepancy in the 'conversation' of a jazz trio. Language \& History, 52, 130-147.

Doffman, M. (2011). Jammin' an ending: Creativity, knowledge, and conduct among jazz musicians. Twentieth-century Music, 8, 203-225.

Doffman, M. (2013). Groove: Temporality, awareness and the feeling of entrainment in jazz performance. In M. Clayton, B. Dueck, and L. Leante (Eds.), Experience and meaning in music performance (pp. 62-85). Oxford: Oxford University Press.

Donald, M. (1990). Origins of the modern mind. Cambridge, MA: Harvard University Press.

Geeves, A., McIlwain, D. J. F., Sutton, J., and Christensen, W. (2014). To think or not to think: the apparent paradox of expert skill in music performance. Educational Philosophy and Theory 46(6), 674-691.

Geeves, A., McIlwain, D. J. F., and Sutton, J. (2014). The performative pleasure of imprecision: a diachronic study of entrainment in music performance. Frontiers in Human Neuroscience, 8(863) October. DOI: 10.3389/fnhum.2014.00863.

Geeves, A., McIlwain, D. J. F., and Sutton, J. (in press). Seeing yellow: connection and 'routine' in professional musicians' experience of music performance. Psychology of Music.

Hutchins, E. (1995). Cognition in the wild. Cambridge, MA: MIT Press.

Hutchins, E. (2010). Cognitive ecology. Topics in Cognitive Science, 2, 705-715.

Keller, P. E. (2008). Joint action in music performance. In F. Morganti, A. Carassa, \& G. Riva (Eds.), Enacting intersubjectivity: A cognitive and social perspective to the study of interactions (pp. 205221). Amsterdam: IOS Press.

Keller, P. E. (2014). Ensemble performance: Interpersonal alignment of musical expression. In D. Fabian, R. Timmers, and E. Schubert (Eds.), Expressiveness in music performance: Empirical approaches across styles and cultures (pp. 260-282). Oxford: Oxford University Press.

Keller, P. E., Novembre, G., and Hove, M. J. (in press). Rhythm in joint action: Psychological and neuropsychological mechanisms for real-time interpersonal coordination. Philosophical Transactions of the Royal Society B. 
Krueger, J. (2011). Doing things with music. Phenomenology and the Cognitive Sciences, 10, 1-22.

Krueger, J. (2014). Affordances and the musically extended mind. Frontiers in Psychology, 4 (1003).

Le Guin, E. (2006). Boccherini's Body: an essay in carnal musicology. Berkeley: University of California Press.

Leman, M. (2007). Embodied music cognition and mediation technology. Cambridge, Mass.: MIT Press.

Leman, M. (2012). Musical entrainment subsumes bodily gestures - its definition needs a spatiotemporal dimension. Empirical Musicology Review, 7, 63-67.

Leman, M., and Maes, P.-J. (2014). Music perception and embodied music cognition. In L. Shapiro (Ed.), The Routledge handbook of embodied cognition (pp. 81-89). Cambridge, MA: MIT Press.

Maes, P.-J., Leman, M., Palmer, C., and Wanderley, M. (2014). Action-based effects on music perception. Frontiers in Psychology, 4 (1008), 1133-1139.

Matyja, J. (2012). Dancing about music, rather than talking about (the musical mind's) architecture: a review of Marc Leman's Embodied Music Cognition and Mediation Technology, AVANT, 3(1), 176179.

Phillips-Silver, J., and Keller, P. (2012). Searching for roots of entrainment and joint action in early musical interactions. Frontiers in Human Neuroscience, 26, 1-11.

Schiavio, A., and Menin, D. (2013). Embodied music cognition and mediation technology: a critical review. Psychology of Music, 41, 804-814.

Sheets-Johnstone, M. (2009). Animation: the fundamental, essential, and properly descriptive concept. Continental Philosophy Review, 42, 375-400.

Sterelny, K. (2010). Minds: extended or scaffolded? Phenomenology and the Cognitive Sciences, 9(4), 465-481.

Sterelny, K. (2012). The evolved apprentice. Cambridge, Mass.: MIT Press.

Sutton, J. (2010). Exograms and interdisciplinarity: History, the extended mind, and the civilizing process. In R. Menary (Ed.), The extended mind (pp.189-225). Cambridge, Mass.: MIT Press.

Sutton, J., Harris, C. B., Keil, P. G., and Barnier, A. J. (2010). The psychology of memory, extended cognition, and socially distributed remembering. Phenomenology and the Cognitive Sciences 9(4), $521-560$

Sutton, J., McIlwain, D. J. F., Christensen, W., and Geeves, A. (2011). Applying intelligence to the reflexes: Embodied skills and habits between Dreyfus and Descartes. JBSP: Journal of the British Society for Phenomenology 42(1), 78-103.

Sutton, J., and Tribble, E. B. (2012). Materialists are not merchants of vanishing. Early Modern Culture, 9. URL: http://emc.eserver.org/1-9/sutton_tribble.html.

Tribble, E. B., and Keene, N. (2011). Cognitive ecologies and the history of remembering: Religion, education, and memory in early modern England. London: Palgrave. 